\title{
¿CÓMO HA RESUELTO EL DESAFÍO DE LA SOBRECARGA DE TRABAJO LA CORTE SUPREMA DE LOS ESTADOS UNIDOS?
}

\author{
HOW HAS THE SUPREME COURT OF THE UNITED STATES SOLVED \\ ITS EXCESSIVE WORKLOAD PROBLEM?
}

\author{
JUAN B. ETCHEVERRY*
}

\begin{abstract}
RESUMEN: La historia de la jurisdicción apelada de la Corte Suprema de los Estados Unidos está, en parte, asociada a la dificultad para resolver la cantidad de casos que recibe cada año. Con este trabajo se busca mostrar cómo, por medio del certiorari, la práctica constitucional de los Estados Unidos soluciona el problema de la sobrecarga de trabajo de su Corte Suprema. Con esta finalidad, se explicará cómo evolucionó y funciona la jurisdicción de la Corte Suprema de los Estados Unidos, qué casos son "potencialmente" admisibles y cuáles "normalmente" rechazados, cómo se tramitan los certioraris y de qué modo se rechazan los no admitidos a trámite. Este esfuerzo se hace a fin de poner de manifiesto que esta solución está orientada por lo que la práctica constitucional de los Estados Unidos considera es la función primordial de su Corte Suprema. Puede ser relevante tener esto en cuenta en los debates que se abren en otros países acerca de cómo enfrentar la sobrecarga de trabajo de sus tribunales de última instancia con competencia para ejercer el control de constitucionalidad.
\end{abstract}

Palabras clave: Certiorari, jurisdicción discrecional, recurso extraordinario, Corte Suprema de los Estados Unidos.

ABSTRACT: The history of the appellate jurisdiction of the United States Supreme Court is, in part, related with the difficulties to decide the volume of cases it receives every year. The purpose of this paper is to show how the incorporation of certiorari solved the excessive workload problem of such court. To achieve this goal, it will be explained how the United States Supreme Court's jurisdiction evolved and how it currently works, which cases could be "potentially" admissible and which are "normally" denied, how this screening process works and how is the way that non-certworthy petitions are denied. This effort is made to show how the certiorari solution is related to what the constitutional practice of United States considers as the main function of its Supreme Court. This explanation could be useful in the context of debates about how to solve excessive workload problems of Courts of last resort that have the power of judicial review.

Key words: Certiorari, discretionary jurisdiction, extraordinary writ, Supreme Court of the United States.

\footnotetext{
Doctor en Derecho por la Universidad de La Coruña (España). Investigador del Consejo Nacional de Investigaciones Científicas y Técnicas de Argentina (CONICET) y profesor de la Universidad Austral (Argentina). Dirección postal: Mariano Acosta 1611(B1629WWA), Pilar, Provincia de Buenos Aires, Argentina. Dirección electrónica: jbetcheverry@austral.edu.ar

Este trabajo forma parte de los resultados de un proyecto de investigación financiado por la Universidad Austral y el Ministerio de Ciencia, Tecnología e Innovación Productiva de Argentina. Debo mi agradecimiento a los profesores Juan Cianciardo, Estela Sacristán, Manuel García Mansilla, Alejandro Uslenghi, Pilar Zambrano, Martín Oyhanarte y Luciano Laise por las críticas ofrecidas a borradores de este trabajo.
} 


\section{PLANTEAMIENTO}

$\mathrm{Al}$ igual que sucede con muchos tribunales de última instancia con competencia para ejercer el control de constitucionalidad ${ }^{1}$, la historia de la jurisdicción apelada de la Corte Suprema de los Estados Unidos está, en parte, asociada a la dificultad para resolver la cantidad de casos que recibe cada año ${ }^{2}$.

Como se verá a continuación, la práctica constitucional de los Estados Unidos afrontó el problema de limitar la cantidad de casos que la Corte Suprema resuelve por año. Para ello, en un primer momento transformó parte de su jurisdicción en discrecional (por medio del instrumento procesal denominado certiorari); para luego terminar circunscribiendo al mínimo o eliminando los otros tipos de su jurisdicción no discrecionales.

Con este trabajo se busca mostrar que dicha solución surge como un modo de asegurar lo que se considera es la función primordial de la Corte Suprema de los Estados Unidos. Este objetivo se persigue con una doble finalidad. En primer lugar, porque el análisis del instrumento procesal del certiorari norteamericano puede ser de utilidad para quienes estudian mecanismos para resolver los problemas de sobrecarga de trabajo de otros tribunales de última instancia con competencia constitucional. En segundo lugar, a fin de poner de manifiesto que en los debates sobre cómo enfrentar la dificultad de la sobrecarga de estos tribunales suele resultar necesario pensar o replantearse cuál es la función primordial de tales tribunales en cada sistema jurídico en particular. Clarificada esta cuestión resulta más sencillo determinar cuál puede ser el mejor modo para que dicho tribunal logre llevar a cabo adecuadamente su función.

Para lograr el objetivo planteado, en primer lugar se explicará cómo surgió y evolucionó la institución del certiorari en la jurisdicción de la Corte Suprema de los Estados Unidos. Luego, se identificarán criterios que permitan distinguir entre casos "potencialmente" admisibles para su resolución y "normalmente" rechazados sin mayor trámite, a fin de advertir la relación que existe entre estos criterios y la finalidad principal que la práctica constitucional de los Estados Unidos le otorga a su Corte Suprema. También se buscará mostrar la vinculación entre dicha finalidad y el modo en que este Tribunal tramita y rechaza los casos que le son presentados año a año. Por último, se hará un balance de la efectividad del certiorari para superar la sobrecarga de trabajo de la Corte Suprema de los Estados Unidos y se advertirán algunos de los desafíos que ha de enfrentar esta solución.

\section{LA INSTAURACIÓN DEL CERTIORARI, LA LIMITACIÓN DEL WRIT OF APPEAL Y LA REFORMA DE LA REGLA 10 REDUCEN LA SOBRECARGA DE TRABAJO}

La jurisdicción de la Corte Suprema de los Estados Unidos está principalmente determinada por el artículo III de la Constitución y por una ley del Congreso. De acuerdo con esta normativa, la Corte tiene jurisdicción sobre 5 tipos de casos. Estos casos son los

\footnotetext{
Le Sueur y Cornes (2000) p. 12.

2 BIANCHI (1994) p. 111.
} 
que puede recibir por: i) certiorari (que son la mayoría de los asuntos que resuelve); ii) jurisdicción originaria; iii) "appeal”; iv) "certification"; y v) "extraordinary writ". Los últimos dos son muy excepcionalmente utilizados. La Corte tiene la obligación de resolver los asuntos que le llegan por el “writ of appeal” y por jurisdicción originaria. La jurisdicción originaria es regida por la segunda sección del artículo III de la Constitución y limitada por la Enmienda XI y versa sobre casos relativos a embajadores, otros ministros públicos y cónsules, así como en aquellos casos en que el conflicto sea entre Estados. Se presentan muy pocos casos de este tipo (solo uno o dos por año). En cambio, la jurisdicción por "appeal" es regida por el Congreso y si bien en algún momento fue voluminosa, desde su reforma en 1988 (por medio de la "Judicial Improvements and Access to Justice Act") prácticamente todas sus categorías han sido eliminadas ${ }^{3}$. Por todo esto, casi la totalidad de los casos que resuelve la Corte Suprema hoy en día le llegan por la vía del certiorari. A diferencia de las anteriores, la jurisdicción del certiorari ha sido concebida como discrecional. Como explica la propia Corte Suprema en su regla 10, la revisión que realiza por una petición de certiorari "no es una cuestión de derecho, sino de discrecionalidad judicial" que se ejercerá "solo por razones imperativas" (compelling reasons) $)^{4}$.

En sus comienzos, prácticamente toda la jurisdicción de la Corte Suprema de los Estados Unidos era obligatoria. Hasta tal punto era de este modo que John Marshall (presidente de dicho Tribunal) llegó a decir (a principios del siglo XIX) que la Corte no tiene ningún derecho a declinar al ejercicio de la jurisdicción que le fuera otorgada, como tampoco lo tiene a usurpar la que no se le diera. A su juicio, una cosa o la otra serían "traicionar la Constitución". La Judiciary Act de 1789 otorgaba jurisdicción a la Corte Suprema estadounidense (por medio del llamado "writ of error") para revisar las sentencias de tribunales federales inferiores sobre casos civiles cuyo monto disputado superara los 2.000 dólares y contra sentencias de tribunales superiores de los estados sobre una cuestión federal ${ }^{6}$. Sumado a esto, se decidió que los jueces de la Corte Suprema dedicasen parte de su tiempo a colaborar en los diversos tribunales federales de circuito que el Congreso fuera creando en territorio de los distintos estados ${ }^{7}$. El problema era que a medida que pasaban los años la Corte, con su doble tarea, se encontraba cada vez más sobrecargada de trabajo. La expansión de Estados Unidos en el siglo XIX hizo colapsar la justicia federal y esto le generó a la Corte muchos años de trabajo atrasado ${ }^{8}$. A modo de ejemplo, en 1890 la Corte

\footnotetext{
3 PeRRY (Jr.) (2005) p. 154.

4 Regla 10 de la Corte Suprema de los Estados Unidos. Resta advertir que esta regla forma parte de un conjunto de reglas que la Corte Suprema de los Estados Unidos tiene potestad para dictar a fin de regular su funcionamiento interno.

5 "[The Supreme Court] had no more right to decline the exercise of jurisdiction which is given, than to usurp that which is not given. The one or the other would be treason to the Constitution". COHENS V. VIRGINIA (1821).

6 Bianchi (1994) pp. 112-117.

7 Si bien Marshall aceptaba que la jurisdicción de la Corte era obligatoria duda de la constitucionalidad de la obligación de los jueces de dicho tribunal de dedicar parte de su trabajo a colaborar en los tribunales federales de circuito. Hobson (2006) p. 1428 y Calabresi y Presser (2006) p. 1394.

8 Hasta la reforma de 1891 la Corte Suprema se rigió por la "Judiciary Act" de 1789. Sobre la historia de la jurisdicción federal y, específicamente, de la Corte Suprema de los Estados Unidos WheEler y Harrinson (2005) pp. 1-4.
} 
Suprema tenía 1.816 casos pendientes de resolución (623 ingresados ese año) y la obligación de resolver todos los casos que le presentaran. Este problema comenzó a solucionarse con la "Circuit Courts of Appeal Act" de 1891 (también conocida como la "Evarts Act"). Esta regulación transfirió muchas de las apelaciones directas a la Corte Suprema hacia los tribunales federales de apelación (llamados Circuit Courts of Appeal), cuyas decisiones en algunos casos podían considerarse finales. Además, dicha regulación redujo notablemente la obligación de los jueces de la Corte de colaborar como jueces de circuito. Sin embargo, la Corte Suprema conservó una parte de su jurisdicción obligatoria y, además, la capacidad para controlar las decisiones de estos tribunales de apelación (introducida por la posibilidad de que se apele a ella por medio del certiorari). Esta reforma transitoriamente permitió aliviar su trabajo, al punto que en 1892 le ingresaron solo 275 casos. Sin embargo, a medida que la Corte Suprema, en las primeras décadas del siglo XX, comenzó a declarar inconstitucionales algunas normativas sociales y económicas nuevamente padeció atraso y sobrecarga de trabajo? .

Recién en 1925 se produjo un importante cambio en la concepción de la jurisdicción de la Corte Suprema ${ }^{10}$. Impulsada por el (ex presidente de los Estados Unidos) Chief Justice William Howard Taft, una comisión conformada por jueces de la Corte Suprema preparó un proyecto de ley presentado al Congreso que transformaba buena parte de la jurisdicción de esta Corte en discrecional. Con esta reforma la Corte Suprema terminó de transferir su poder de revisor de los asuntos de derecho federal a los tribunales de circuito y se transformaba en un árbitro de asuntos judiciales de importancia nacional ${ }^{11}$. Triunfaba entonces la visión de Taft que consideraba que la única manera de resolver el problema de la sobrecarga de trabajo de una vez y para siempre era transformar casi toda la jurisdicción de la Corte Suprema en discrecional. Taft era consciente de que su propuesta implicaba para la Corte una importante pérdida de funciones. Sin embargo, debido al crecimiento de la cantidad de casos que recibía, defendía que era el único modo de lograr asegurar lo que consideraba la principal función de la Corte: "no preservar los derechos de los litigantes", sino "exponer y estabilizar los principios jurídicos" y preservar la "uniformidad de las decisiones entre los tribunales intermedios de apelación"12. En la reforma de 1988 se terminó de eliminar prácticamente todo lo que quedaba de la jurisdicción obligatoria de la Corte, simplemente enmendando la reforma de 1925 y regularizando lo que, para ese entonces, la práctica de la propia Corte ya en buena medida había impuesto: no considerar a las apelaciones que no justificasen una revisión por certiorari ${ }^{13}$. Dicha regularización fue llevada a cabo por medio de una reforma de las leyes que regulan su jurisdicción y de algunas de sus reglas internas (especialmente la regla 10$)^{14}$.

\footnotetext{
9 Bianchi (1994) pp. 117-118.

10 Sternberg pone énfasis en el quiebre radical que se produce en el modo en que se entiende la jurisdicción de la Corte Suprema de los Estados Unidos a partir de la Judiciary Act de 1925. STERnBerg (2008) p. 2.

11 BiAnCHI (1994) p. 119.

12 Hartnett (2000) pp. 1664-1665.

13 Sternberg (2008) p. 13.

14 Perry (Jr.) (2005) p. 154.
} 
Conforme a esto último, resulta inevitable y de gran importancia preguntarse: ¿con qué criterios decide la Corte Suprema de los Estados Unidos qué casos justifican una revisión por certiorari? Si bien las principales leyes sobre la jurisdicción de la Corte Suprema (secciones 1254(1) y 1257 del Código Federal de los Estados Unidos) y la mencionada regla 10 ofrecen algunos criterios por los que un caso puede ser admitido para revisión, en realidad la decisión parece tener condimentos subjetivos o de “intuición”. Tanto es así que el propio ex juez de la Corte William H. Rehnquist llegó a afirmar que la decisión de aceptar o no un certiorari es una "decisión bastante subjetiva, en parte compuesta de intuición y en parte de razonamiento jurídico" 15 .

A continuación esbozaremos algunos de los criterios que la doctrina ha intentado reconstruir para quienes pretenden litigar ante la Corte.

\section{LAS REGLAS, LOS CASOS QUE SON "POTENCIALMENTE" ADMISIBLES Y LOS QUE SON “NORMALMENTE” RECHAZADOS}

Las reglas recogidas en las secciones 1254(1) y 1257 del título 28 sobre "Procedimientos Judiciales” (parte IV, “jurisdicción”, capítulo 81, “Corte Suprema”) del Código Federal de los Estados Unidos y la regla 10 del reglamento de la Corte Suprema de los Estados Unidos describen en líneas generales los tipos de casos que provienen de tribunales federales de apelación o de tribunales superiores de los estados que potencialmente podrían ser admitidos para su resolución por medio del certiorari. En concreto, la sección 1254(1) extiende ampliamente la jurisdicción del certiorari a casi todos los casos civiles o penales que se encuentren en las cortes federales de apelación. En cambio, de un modo que circunscribe mucho más el certiorari, la sección 1257 faculta a la Corte a revisar las decisiones finales otorgadas por los tribunales superiores de los estados en las que la validez de una ley federal o local sea cuestionada por ir contra el derecho federal o cuando un derecho se encuentra especialmente fundado en el derecho federal ${ }^{16}$. Por otra parte, los criterios que ofrecen estas normas deben ser interpretados a la luz de la regla 10 de la Corte Suprema de los Estados Unidos que (como anteriormente explicamos) establece que la revisión por certiorari no es una cuestión de derecho, sino de discrecionalidad que se ejercerá solo por razones imperativas. Además, a modo de ejemplo, también aclara que estas razones se pueden dar cuando: i) surgen conflictos entre decisiones de diferentes tribunales federales de apelación sobre un mismo tema "importante"; ii) aparecen conflictos sobre una "cuestión federal importante" por lo decidido entre tribunales federales de apelación y tribunales superiores de los estados o simplemente entre tribunales superiores de los estados; iii) una decisión de una corte federal de apelación decide sobre una "cuestión importante de derecho federal" que no está determinada y debería estarlo por la Corte Suprema o la decisión de la corte de apelación sobre una "cuestión importante de derecho federal" entra en conflicto con decisiones relevantes de la Corte Suprema. También puede excepcionalmente intervenir cuando

15 RehnQuist (1987) p. 265.

16 Se ha tenido acceso al Código Federal de los Estados Unidos por medio de: 〈http://uscodebeta.house.gov/ browse/prelim@title28/part4/chapter81\&edition=prelim> 
una Corte de Apelaciones Federal se separa del curso usual y aceptado de los procedimientos judiciales, aunque raramente invoca esta causal. Por último, resta advertir que expresamente se aclara que es muy raro que se acepte un certiorari por errores sobre los hechos del caso o sobre la aplicación de una norma adecuadamente sancionada ${ }^{17}$.

Al margen de estos criterios reglados, debido a que el writ of certiorari es discrecional, para establecer cuándo una petición de certiorari es o no "potencialmente" admisible ha de tenerse en cuenta, ante todo, la práctica de la propia Corte Suprema al respecto ${ }^{18}$. La doctrina ha realizado muchas clasificaciones posibles de estos criterios jurisprudenciales ${ }^{19}$. Para que la síntesis que se ofrecerá sea lo más clara posible, se agruparon dichos criterios en 3 subgrupos: criterios generales, criterios por medio de los cuales una petición de certiorari es normalmente rechazada sin mayor trámite y criterios para que una petición de certiorari sea "potencialmente" admisible para su resolución ${ }^{20}$.

\section{A. Criterios generales}

i. Prioridad del problema sobre el caso: Debido a que la Corte considera que su papel institucional no es enmendar errores de otros tribunales, sino clarificar el derecho: "[para dicho tribunal] lo importante no son los casos, sino las cuestiones o problemas que surgen de esos casos" ${ }^{21}$. Este criterio está vinculado con el siguiente y explica por qué muchos jueces y letrados de la Corte Suprema consideran que "nunca es un error rechazar una petición de certiorari, solo es un error aceptar una [petición] inadecuada”. Esta afirmación

17 La regla 10 establece específicamente que: "Review on a writ of certiorari is not a matter of right, but of judicial discretion. A petition for a writ of certiorari will be granted only for compelling reasons. The following, although neither controlling nor fully measuring the Court's discretion, indicate the character of the reasons the Court considers: (a) a United States court of appeals has entered a decision in conflict with the decision of another United States court of appeals on the same important matter; has decided an important federal question in a way that conflicts with a decision by a state court of last resort; or has so far departed from the accepted and usual course of judicial proceedings, or sanctioned such a departure by a lower court, as to call for an exercise of this Court's supervisory power; (b) a state court of last resort has decided an important federal question in a way that conflicts with the decision of another state court of last resort or of a United States court of appeals; (c) a state court or a United States court of appeals has decided an important question of federal law that has not been, but should be, settled by this Court, or has decided an important federal question in a way that conflicts with relevant decisions of this Court. A petition for a writ of certiorari is rarely granted when the asserted error consists of erroneous factual findings or the misapplication of a properly stated rule of law".

18 Perry sostiene que en alguna medida las reglas sobre el certiorari son tautológicas porque establecen que un caso ha de ser revisado por la Corte Suprema cuando es lo suficientemente importante y esto ocurre cuando sus jueces consideran que un caso es importante. Perry (Jr.) (1994) p. 221.

19 Un repaso de dichas posibles clasificaciones puede verse en STERN et al. (1986) p. 196.

20 Somos conscientes de que según como presentemos un criterio, en sentido positivo o negativo, puede ser clasificado como capaz de establecer la admisibilidad potencial de un pedido o para establecer su normal rechazo. De igual modo, también consideramos que algunos de estos últimos criterios se derivan claramente de los criterios generales. Sin embargo, creemos que la clasificación sirve para hacer más clara la explicación del funcionamiento de la Corte sobre el punto que nos interesa.

21 Existen algunas excepciones a este principio que la doctrina ha denominado los casos indignantes (egregious). Perry (Jr.) (1994) p. 220 y cap. 4. 
es defendida con el argumento de que si un problema es realmente importante, volverá a plantearse ${ }^{22}$.

ii. Presunción de inadmisibilidad: "Las peticiones tienen una presunción de inadmisibilidad". Esta regla se manifiesta en el hecho de que alrededor del $95 \%$ de las peticiones de certiorari para que la Corte revise un caso son denegadas y en que, a fin de lograr reducir la cantidad de casos que la Corte admite por año para su resolución, sus jueces fomentan la siguiente serie de reglas informales entre sus letrados y asistentes: "todo caso que puede ser evitado, ha de ser rechazado"; "el trabajo de los letrados de la Corte Suprema es encontrar razones para denegar certioraris"; "en caso de duda debe optarse por el rechazo"; "un memorándum sugiriendo la aceptación de un certiorari ha de mostrar por qué este caso es uno de los casos más importantes del año"; etc ${ }^{23}$.

\section{B. Criterios con los Que normalmente es rechazada una petición de CERTIORARI}

iii. Aplicación de derecho local por un tribunal federal: Es muy raro que la Corte acepte un certiorari para revisar la aplicación de derecho local (estadual) por un tribunal federal de apelación ${ }^{24}$.

iv. Aplicación de derecho local por un tribunal no federal: La Corte nunca acepta un certiorari para revisar la aplicación de derecho local por un tribunal local de apelación por más que existan conflictos entre las decisiones de dichos tribunales. Estos casos suelen ser denominados por los letrados de la Corte como "frivolous". Dichos letrados suelen estar atentos a que un caso de este tipo no sea disfrazado de un problema de derecho federal ${ }^{25}$.

v. Problemas vinculados con hechos muy particulares: La Corte, en principio, no acepta para su resolución casos con hechos lo suficientemente particulares o complejos (bad facts) que no sirvan para extraer un principio jurídico que permita clarificar al derecho en general $^{26}$.

vi. Discusión sobre hechos o prueba: La Corte, en principio, no acepta pedidos de certiorari en los que se pretende discutir los hechos de un caso o cuestiones de prueba porque no sirven como un buen vehículo (good vehicule) para clarificar el derecho ${ }^{27}$.

22 Perry (Jr.) (1994) p. 221.

23 Shapiro (1998) p. 27 y Perry (Jr.) (1994) p. 219.

24 SHAPIro (1998) p. 27.

25 Perri (Jr.) (1994) p. 222.

26 Perry (Jr.) (1994) p. 234.

27 Perry (Jr.) (1994) p. 234 y Shapiro (1998) p. 27. 
vii. Discusión sobre la aplicación del derecho a un caso: La Corte no suele aceptar los pedidos que tratan sobre la aplicación del derecho a unos hechos y circunstancias muy específicas o particulares, basándose en la regla 10 que rige su funcionamiento. Sin embargo, excepcionalmente admite casos de este tipo en los que está en juego el derecho a que las investigaciones y detenciones realizadas por una autoridad del gobierno no sean arbitrarias (4ta enmienda) y a fin de demostrar la correcta aplicación del derecho a situaciones fácticas recurrentes ${ }^{28}$.

viii. Problemas sin antecedentes sobre cómo ser resueltos: La Corte suele rechazar los casos que no se encuentran lo suficientemente filtrados, decantados o probados por las resoluciones dadas por otros tribunales (percolated), de forma que cuando ofrezca su interpretación final, esta sea lo más clara posible y se pueda beneficiar de la experiencia obtenida por otras resoluciones ${ }^{29}$.

ix. Problemas para los que la Corte no tiene una solución relativamente uniforme: Algunos autores han sugerido que un caso puede ser rechazado porque se presenta como de muy difícil resolución (intractableness) o porque la Corte no sabe mínimamente cómo podría resolverlo o si su resolución exigiera que la Corte divida mucho sus opiniones ${ }^{30}$.

\section{Criterios para Que una petición de CERTiorari SEA “potencialmente” ACEPTABLE:}

x. Circuit conflict: En la actualidad, los abogados están acostumbrados a realizar una petición de certiorari cuando existe un conflicto (circuit conflict) entre las decisiones de la Corte Suprema, los tribunales federales de apelación, los tribunales superiores de los estados y las cortes de apelación militar sobre una cuestión de derecho federal o de derecho general y cuya jurisprudencia la Corte Suprema puede uniformar ${ }^{31}$. Sin embargo, como se ha visto en los criterios v a x, la existencia de estos conflictos no garantiza la aceptación del certiorari porque puede que el caso no esté suficientemente filtrado (percolated), contenga hechos (bad facts) que dificultan que el caso sea un buen vehículo (good vehicule) para clarificar el derecho, o que la petición no tenga ninguna importancia social, o sea de muy difícil resolución (intractable) ${ }^{32}$.

xi. Cuestión importante: Una petición de certiorari es potencialmente aceptable no solo por permitir resolver un conflicto en la jurisprudencia sobre un tema, sino también por tratar sobre una cuestión o problema importante (important issue). De hecho, un problema puede ser lo suficientemente importante como para generar un caso admisible inclu-

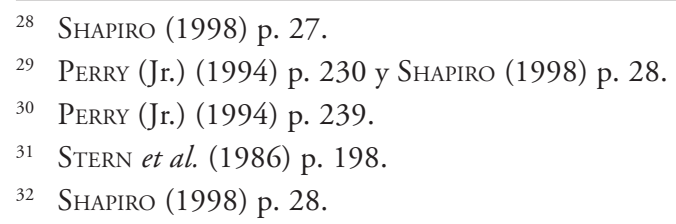


so si no hay un conflicto en la jurisprudencia. Además, una petición para revisar un caso sobre cuya resolución existe conflicto de jurisprudencia puede ser rechazada porque afecta solo a unas pocas personas y no tienen una importancia que impacte en la sociedad ${ }^{33}$. No obstante, los problemas importantes suelen generar conflictos. La dificultad es establecer qué es una cuestión importante. La doctrina ha clasificado los casos importantes de muchos modos. Uno de dichos modos sostiene que hay tres tipos de casos importantes. En primer lugar, los casos que son "importantes en sí mismos y más allá de ellos" (important in and of themselves) y para los cuales no rige la regla del filtrado, de los hechos simples o del buen vehículo para clarificar el derecho. Un ejemplo de este tipo de caso es U.S. v. Nixon, en el que la Corte rechaza el alegato de privilegio e inmunidad del poder ejecutivo. En segundo lugar, existen los casos que son "importantes política y socialmente" (important to the polity). Un ejemplo de este tipo de casos es Brown v. Board of Education, sobre segregación racial en las escuelas. Por último, también pueden mencionarse los casos "importantes para el derecho". Ejemplos de este tipo de casos pueden ser la clarificación de un procedimiento administrativo o de una regla sobre la prueba ${ }^{34}$. Un caso de este tipo sería Goldberg v. Kelly, sobre audiencia previa a la decisión administrativa.

\section{TRÁMITE: EL SISTEMA DE CERTIORARI POOLS, LA LISTA DE DISCUSIÓN, LA CONFERENCIA Y LA NO MOTIVACIÓN DE LOS RECHAZOS}

El trámite interno para tomar la decisión de aceptar o no una petición de certiorari refleja bastante bien la concepción que tiene la propia Corte Suprema de su función principal. Dicho trámite comienza con la recepción del pedido que puede ser entregada en la oficina del auxiliar letrado de la Corte (Clerk of the Court) o enviada por mensajero o correo ${ }^{35}$. Esta oficina cumple la función de primer filtro para las peticiones de certiorari al fiscalizar que dichas peticiones cumplan con los plazos y requerimientos técnicos exigidos en las reglas de la Corte Suprema ${ }^{36}$. De la Clerk's Office las peticiones son llevadas a los despachos (Chambers) de los Jueces y a sus Law Clerks (cada Justice cuenta con cuatro de ellos, salvo el Chief Justice que cuenta con cinco) para el primer estudio (no meramente formal) de los asuntos.

Para poder seleccionar de modo eficiente las peticiones de certiorari que serán aceptadas y separar las que serán rechazadas, ocho de los nueve jueces han formado un grupo de trabajo conjunto que divide entre sus miembros la tarea de realizar memorándums sobre

\footnotetext{
33 Shapiro (1998) p. 27.

34 Perry (Jr.) (1994) p. 253.

35 No debe confundirse el Clerk of the Court con los Law Clerk to the Justices que son los secretarios letrados de la Corte Suprema. Las distintas funciones de los distintos Clerks en la Corte Suprema de los Estados Unidos se encuentran explicados en STERN et al. (1986) pp. 13-21.

36 La regla 12 establece la cantidad de copias que hay que presentar del pedido, la tasa (ver también la regla 38) y el modo en que debe presentarse. La regla 13 otorga 90 días para presentar el certiorari y la 14 un listado de contenidos (y de su orden) de la petición. La regla 33 (entre otras cosas) fija características físicas del pedido (como el número de páginas, dimensiones, etc.).
} 
la totalidad de las peticiones. Esta práctica de trabajo en equipo de los Law Clerks de los distintos Justices de la Corte Suprema ha sido denominada certiorari pool ${ }^{37}$. Cada equipo de Law Clerks revisa entonces una octava parte de la totalidad de los certioraris y prepara una memoria (certiorari pool memo) que es distribuida entre el resto de Law Clerks de los otros Justices. Luego, otro Clerk examina las memorias recibidas y si lo considera necesario la anota o completa para su Justice (mark up memo). Con esto se pretende evitar la posibilidad de que algún Clerk manipule la decisión de la admisión o no de un pedido de certiorari por medio de sus memorias ${ }^{38}$. Todo este material (los documentos del caso, la memoria y las anotaciones o ampliaciones de ellas) es enviado a los Justices. En general, ellos solo tienen en cuenta las memorias para decidir, salvo que surjan dudas sobre qué decisión tomar. Si bien en muchos casos los Justices no estudian directamente los documentos originales, cada uno de ellos está obligado a conocer cada uno de los casos que llegan a su jurisdicción porque su potestad de juzgar no puede ser delegada ${ }^{39}$.

Los jueces que no han adherido al cert. pool han de evaluar cada petición de certiorari. En la actualidad, solo el Justice Alito no es parte del certiorari pool ${ }^{40}$. En este caso, todas las peticiones de certiorari que llegan son divididas entre los Clerks de dicho Justice que elaboran memorias exclusivas para el juez para el que trabajan. Tales memorias tienen básicamente la misma información que las que se elaboran en el cert. pool., salvo que suelen ser más cortas y menos formales.

Redactadas las memorias y realizadas las anotaciones y notas complementarias (mark up) el presidente de la Corte (Chief Justice) prepara (basándose principalmente en las memorias y sus anotaciones) una lista de discusión (discuss list) que contiene los casos que considera que merecen la pena ser discutidos en el plenario (conference). Luego, esa lista se circula entre los otros ocho jueces. Cada Justice puede solicitar al funcionario administrativo del Chief Justice agregar casos para su discusión. Nuevamente todos son informados de estas incorporaciones ${ }^{41}$.

Todos los casos que no son incorporados en esta lista de discusión quedan automáticamente rechazados, sin que siquiera se haga referencia a ellos en el plenario. Simplemente, los casos que no figuran en la lista de discusión pasan a una nueva lista, denominada eufemísticamente como dead list, dándose a conocer como certioraris rechazados por medio de la lista del lunes siguiente al viernes de plenario (Monday order list). Además, el Clerk de la Corte, notifica por carta a los abogados. Solo excepcionalmente pueden intentar que un caso que no figuraba en la lista pueda ser introducido en la lista de discusión del siguiente plenario (relisting). Suele alegarse en estos casos que no se tenía toda la información para decidir.

\footnotetext{
7 Sobre el certiorari pool puede verse STURLEY (2005) pp. 155-156.

38 Con dicha finalidad suelen contratarse como Clerks abogados recién graduados que, además, no suelen durar más de un año en su cargo. Sobre la evolución y el debate acerca del rol de los Law Clerks en la Corte Suprema de los Estados Unidos cfr. Peppers (2006) passim.

39 STERn et al. (1986) p. 257.

40 LIPTAK (2008).

41 STERN et al. (1986) p. 259.
} 
Finalmente, en las reuniones plenarias de los viernes (Friday conferences) fijadas a principio del año, los nueve jueces discuten y votan sobre la aceptación o no de los pedidos de certiorari que están en la lista elaborada para dicha reunión. La sesión no es pública ni abierta a otros miembros del equipo de Tribunal. El Chief Justice presenta brevemente los hechos del caso, las cuestiones controvertidas y da su opinión. Luego el resto de los jueces, por orden de antigüedad, opinan y anuncian su voto. Para que un certiorari sea admitido y el caso tratado debe obtener, al menos, cuatro votos favorables (Rule of four). Una vez votado se publican las listas de los certioraris admitidos y de los rechazados y el Clerk del Tribunal notifica esto ${ }^{42}$.

\section{PARA RESOLVER LA SOBRECARGA DE TRABAJO DE LA CORTE SUPREMA FUE NECESARIO ACLARAR O REPLANTARSE SU FUNCIÓN PRINCIPAL}

La utilización del certiorari como herramienta para la solución de la sobrecarga de trabajo de la Corte Suprema de los Estados Unidos comenzó con la reforma de la Judicial Act de 1925 promovida por el ex presidente de los Estados Unidos y, en ese momento, presidente de la Corte Suprema William Howard Taft. La propuesta de Taft suponía un cambio radical en la jurisdicción de la Corte Suprema e, inicialmente, generó una fuerte resistencia entre algunos jueces (de diferentes instancias), senadores e incluso por la American Bar Association. Sin embargo, la promesa del bajo costo que suponía implementar la reforma, la solución que ofrecía a los problemas de retraso que tenía la Corte, la determinación de ser una solución definitiva frente a las otras propuestas de solución del problema de la sobrecarga de trabajo y el peso político de Taft terminaron haciendo que su propuesta fuera transformada en ley en febrero de 1925 y casi sin discusión en las Cámaras ${ }^{43}$.

En la actualidad, la mayor parte de la doctrina y la práctica de la propia Corte $\mathrm{Su}$ prema de los Estados Unidos concuerdan en que la principal función de dicho Tribunal es interpretar y aclarar el derecho. En otras palabras, ya ha sido casi unánimemente aceptado que la función primordial de la Corte Suprema de los Estados Unidos es ofrecer una interpretación final del derecho federal ${ }^{44}$. Esta definición tan clara del papel de este Tribunal pareciera que es lo que ha permitido que se aborde con mucha decisión el establecimiento de un procedimiento que le permita cumplir con dicho papel.

Tal procedimiento otorga discrecionalidad a la Corte Suprema para seleccionar los casos que acepta e, incluso, le permite rechazar las peticiones de certiorari sin ningún tipo de motivación o argumentación. Dicho de otro modo, en lo que respecta a los certioraris, los jueces son libres de votar por su aceptación o rechazo sin ofrecer razones para su deci$\operatorname{sió}^{45}$. No obstante, ha de reconocerse, que se publican todas las peticiones de certiorari,

42 STERN et al. (1986) pp. 260-264.

43 Sternberg (2008) pp. 11-12.

44 Perry (Jr.) (1994) p. 230.

45 Existen excepciones al respecto. En algunos casos la Corte de modo breve e ilustrativo explica cuál ha sido la causal del rechazo del pedido de Certiorari. Justice Stevens argumentó -en CoUNTY OF SONOMA V. ISBELL (1978) e ILLINOIS V. GRAY (1978)- que tal práctica era incoherente con la regla de que los rechazos no tienen valor como precedente. STERn et al. (1986) p. 267. 
al igual que la inmensa mayoría de las sentencias apeladas. Por lo tanto, es posible conocer qué casos se admiten y cuáles no ${ }^{46}$. Aunque, como se ha advertido, el rechazo de un certiorari no tiene significado ninguno ni resulta vinculante para el tribunal ${ }^{47}$. Más aún, para la Corte Suprema "nunca es un error rechazar una petición de certiorari, solo es un error aceptar una inadecuada", porque considera que si un problema es realmente importante, volverá a plantearse. Además, como se ha visto con el método de filtrado (percolation) que utiliza la Corte, puede que un caso resulte importante, pero que no sea aceptado simplemente porque se está esperando que instancias inferiores traten el tema para tener más información para decidir un caso similar más adelante.

Por esta misma razón, la Corte prioriza la resolución de una cuestión de derecho federal que necesite ser clarificada sobre la resolución de un caso o controversia particular. Así, en principio, se admiten para resolución solo aquellos casos en los que existe un conflicto entre tribunales de apelación federal o superiores tribunales sobre cómo entender al derecho federal, cuyos hechos son claros o sirven como un buen vehículo para clarificar dicho derecho, o porque tratan sobre una cuestión o problema importante. A su vez, por todo esto, no se admiten casos en los que se discuten solo hechos o pruebas, o casos que tratan sobre la aplicación del derecho a unos hechos y circunstancias particulares, o casos que resultan de muy difícil resolución (sea porque la Corte no sabe mínimamente cómo podría resolverlos o porque su resolución exigiría que la Corte divida mucho sus opiniones), o casos en los que existe un conflicto de jurisprudencia cuya resolución afecta solo a unas pocas personas y que carece de una importancia que impacte en la sociedad.

De hecho, todo el proceso por el cual se tramitan internamente los certioraris, descrito en el epígrafe anterior, parece ser un mecanismo de filtros en etapas (desde la recepción de las peticiones, pasando por las memorias elaboradas por los letrados, las anotaciones de dichas memorias, las listas de discusión y las de descarte, hasta llegar a la discusión y votación) que permite a los jueces descartar buena parte de las peticiones que reciben, a fin de que puedan concentrarse únicamente en las cuestiones que consideran importantes ${ }^{48}$.

En definitiva, la institucionalización del certiorari parece haber dado sus frutos. La Corte Suprema de los Estados Unidos resuelve solo entre 70 u 80 casos por año y casi no tiene retraso en su trabajo. Con la participación casi unánime de los jueces en el certiorari pool, la Corte Suprema ha logrado decidir el rechazo o la aceptación de las aproximadamente 9.000 peticiones que recibe anualmente ${ }^{49}$. Como sugiere Shapiro, ha diseñado una línea defensiva capaz de "repeler legiones de peticiones". Por ello, pocos son los autores que hoy consideran que la Corte Suprema está sobrecargada de trabajo. Por el contrario, surgen nuevas voces que ponen en duda el hecho de que la Corte esté resolviendo "suficientes casos importantes" $"$.

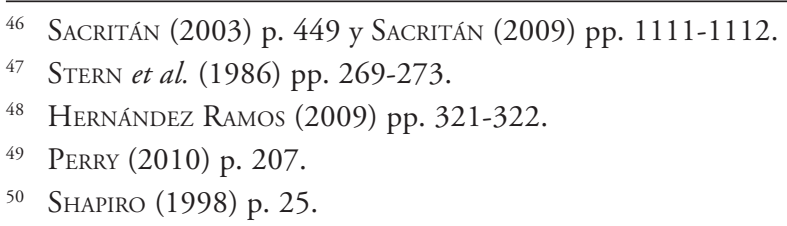


De la experiencia de la práctica constitucional norteamericana sobre cómo enfrentó la sobrecarga de trabajo de su Corte Suprema puede advertirse que, para abordar tal dificultad, le resultó necesario pensar y replantearse cuál era la función primordial de dicho tribunal y decidir concentrarse solo en ella. Clarificada esta cuestión fue más sencillo determinar cuál era el mejor modo para que logre llevar a cabo dicha función.

\section{DESAFÍOS Y ALTERNATIVAS POSIBLES A LA PRÁCTICAMENTE ILIMITADA JURISDICCIÓN DISCRECIONAL DE LA CORTE SUPREMA}

En el contexto anteriormente descripto se empiezan a escuchar con más fuerza voces críticas sobre la "casi ilimitada discrecionalidad" de la que goza, o sobre la combinación de casos que la Corte Suprema de los Estados Unidos ha aceptado en los últimos $\operatorname{casos}^{51}$, o en razón de los pocos casos que cada año escucha ${ }^{52}$, o por su excesiva confianza en la tarea de sus secretarios letrados ${ }^{53}$. El problema es que hoy en día la Corte Suprema se juega buena parte de su prestigio, imagen, autoridad política (y moral) y buena parte del alcance de su papel institucional en la decisión de qué casos aceptar ${ }^{54}$. Por ello, en los últimos años se han publicado algunos trabajos repensando la institución del certiorari o, por ejemplo, iluminando la discrecionalidad con la que opera la Corte Suprema para seleccionar los casos que acepta con principios del Derecho Administrativo estadounidense ${ }^{55}$.

Tener en cuenta estos desafíos también puede ser de interés para quienes estudian cómo la Corte Suprema de los Estados Unidos resolvió su problema de exceso de trabajo (transformando casi toda su jurisdicción en discrecional por medio de la institución del certiorari), a fin de buscar mecanismos de solución para la sobrecarga de trabajo de otros tribunales de última instancia con competencia para ejercer el control de constitucionalidad. Dicho de otro modo, también puede resultar útil para las prácticas constitucionales que evalúan la incorporación o el modo de implementación del certiorari como herramienta para superar el problema de la sobrecarga de trabajo de sus tribunales de última instancia, tener en cuenta los desafíos que en los últimos años se le han planteado a este tipo de solución.

En este sentido, resulta particularmente interesante analizar algunas formas concretas de restringir de algún modo o en alguna medida la discrecionalidad con que se aceptan o rechazan para su revisión los recursos. A continuación expondremos dos alternativas que parecen capaces tanto de limitar en alguna medida la discrecionalidad con que se eligen los casos a tratar, como de evitar que se caiga o recaiga en un problema de sobrecarga de trabajo. Si bien exigirle a un máximo tribunal que justifique todos los rechazos de los recursos

51 TYLER (2009) p. 1310.

52 WatTs (2011) p. 1.

53 Carrington y Cramton (2009) p. 591.

54 Meriwether Cordray y Cordray (2004) p. 397; Meriwether Cordray y Cordray (2008) p. 1 y Meriwether Cordray y Cordray (2009) pp. 314-315.

55 Watts (2011) passim. Más recientemente, William Baude ha criticado lo oscuros que resultan los criterios con que la Corte Suprema de los Estados Unidos selecciona los casos que acepta para su tratamiento cada año. BAUde (2015) p. 3 ss. 
que se le interponen (como suele exigírsele a tribunales de instancias inferiores) puede generar (nuevamente) un problema de sobrecarga de trabajo, una práctica que podría adoptar o exigírsele a dicho tribunal es que, al menos, explicite el criterio que utiliza para aceptar un caso para su tratamiento. De este modo, se vuelve más previsible cuándo un recurso es posible que sea aceptado para su tratamiento. Además, el tribunal se puede ver en alguna medida constreñido por sus propios antecedentes de aceptación de un tipo de caso. Todo esto puede ayudar a que se vuelva más transparente el obrar de estos tribunales de forma tal que no quede restringido el conocimiento de cuándo es probable o no que un caso sea aceptado para su tratamiento a una elite de abogados especializados en cómo funcionan tales tribunales (como algunos doctrinarios denuncian que sucede en Estados Unidos) ${ }^{56}$.

Más allá de estas críticas sobre cómo funciona la jurisdicción apelada de la Corte Suprema de los Estados Unidos, hay que reconocer que tanto la legislación y principalmente la doctrina especializada se esfuerzan por ir develando los criterios con los que la Corte Suprema decide aceptar o rechazar un caso para su tratamiento. Además, la propia Corte Suprema de los Estados Unidos se muestra proclive a brindar información sobre tales criterios $^{57}$. Como muestra de esto puede ser útil recordar, entre otras cosas, que si bien la Corte Suprema de los Estados Unidos normalmente no funda la no aceptación de un caso para su tratamiento, excepcionalmente sí lo hacen algunos de sus jueces, por ejemplo, cuando no comparten la decisión que tomó la mayoría del Tribunal. Otro ejemplo que ilustra cómo la Corte Suprema de los Estados Unidos es permeable a brindar información sobre los criterios que utiliza para aceptar o rechazar un caso para su tratamiento ha sido la autorización que la Corte le dio al profesor Perry para entrevistar a muchos de sus funcionarios letrados y jueces a fin de preguntarles sobre esta cuestión. En esta oportunidad se impuso como única limitación para la publicación de dichas entrevistas, que no se revelara quién hizo cada una de las afirmaciones recogidas en ellas, aunque se permitió que se dijera cuál era el cargo de las personas cuyas declaraciones finalmente se publicaron. El resultado de dicha interesante investigación se publicó en un libro titulado Deciding to Decide. Agenda Setting in the United States Supreme Court ${ }^{58}$.

Otra alternativa para limitar un poco la discrecionalidad de los tribunales de última instancia para elegir los casos que tratarán, aunque sin eliminarla por completo, es la adoptada por la Ley Orgánica del Tribunal Constitucional de España (de ahora en adelante, LOTC). Dicha ley no le otorga expresamente al Tribunal Constitucional la potestad de seleccionar libre o discrecionalmente qué recursos de amparo ha de tratar y cuáles ha de rechazar. En cambio, constriñó al Tribunal Constitucional a admitir los recursos que satisfagan los criterios establecidos legalmente. No obstante, la formulación de dichos criterios es abstracta, vaga y requiere de determinación judicial. Más aún, luego de la reforma de 2007, la LOTC exige para la aceptación de un amparo, entre otras cosas, que el caso justifique "una decisión sobre el fondo por parte del Tribunal Constitucional en razón de su especial

\footnotetext{
56 Algunos autores sugieren que el procedimiento de aceptación de peticiones de certiorari ha generado que una elite de abogados expertos en cómo funciona la Corte Suprema sea la que logre buena parte de dichas aceptaciones. LAZARUS (2009) p. 89.

57 Sotomayor es el juez que ha utilizado en más ocasiones este recurso. Al respecto YABLON (2014) pp. 551 ss.

58 Perry (2010) passim.
} 
trascendencia constitucional” (art. 50.1.b). Si bien, la LOTC no otorga explícitamente al Tribunal Constitucional discrecionalidad para la selección de los casos que considera "constitucionalmente trascendentes", varios autores advierten que la decisión de admitir o no una demanda de amparo conforme a este diseño es en la práctica muy discrecional ${ }^{59}$. En este contexto, el propio Tribunal Constitucional de España ha decidido ir aclarando en los últimos años qué entiende por casos de "especial trascendencia constitucional". Con particular empeño ha realizado esta tarea en la sentencia STC 155/2009 al enumerar siete supuestos en los que se daría dicha especial trascendencia ${ }^{60}$. Con esta sentencia el Tribunal Constitucional determina y dota de contenido al concepto de "especial trascendencia constitucional" ${ }^{1}$. No obstante, esta determinación no es definitiva ni cerrada pudiendo, según las circunstancias y por medio de las decisiones del tribunal, ampliarse o reducirse. El propio tribunal es consciente y expresa esto mismo al afirmar que los siete supuestos enunciados no pueden ser entendidos como "un elenco definitivamente cerrado de casos en los que un recurso de amparo tiene especial trascendencia constitucional", porque esto se opone al "carácter dinámico" de su jurisdicción que necesita muchas veces a partir de la casuística "perfilar o depurar conceptos, redefinir supuestos contemplados, añadir otros nuevos o excluir alguno inicialmente incluido" ${ }^{2}$. En definitiva, por más que la aclaración que hace sirve como modo de autolimitación, el dinamismo que el tribunal le reconoce a los supuestos que enumera y el modo en que han sido enunciados algunos de ellos, no eliminan el

59 García Roca (2003). Sobre el debate previo a la reforma en relación a este punto puede verse CARMONA Cuenca (2005) pp. 111 ss.

60 Así, en el fundamento jurídico $2^{\circ}$ expresa que dichos casos serán:

“a) el de un recurso que plantee un problema o una faceta de un derecho fundamental susceptible de amparo sobre el que no haya doctrina del Tribunal Constitucional, supuesto ya enunciado en la STC 70/2009, de 23 de marzo; b) o que dé ocasión al Tribunal Constitucional para aclarar o cambiar su doctrina, como consecuencia de un proceso de reflexión interna, como acontece en el caso que ahora nos ocupa, o por el surgimiento de nuevas realidades sociales o de cambios normativos relevantes para la configuración del contenido del derecho fundamental, o de un cambio en la doctrina de los órganos de garantía encargados de la interpretación de los tratados y acuerdos internacionales a los que se refiere el art. 10.2 CE; c) o cuando la vulneración del derecho fundamental que se denuncia provenga de la ley o de otra disposición de carácter general; d) o si la vulneración del derecho fundamental traiga causa de una reiterada interpretación jurisprudencial de la ley que el Tribunal Constitucional considere lesiva del derecho fundamental y crea necesario proclamar otra interpretación conforme a la Constitución; e) o bien cuando la doctrina del Tribunal Constitucional sobre el derecho fundamental que se alega en el recurso esté siendo incumplida de modo general y reiterado por la jurisdicción ordinaria, o existan resoluciones judiciales contradictorias sobre el derecho fundamental, ya sea interpretando de manera distinta la doctrina constitucional, ya sea aplicándola en unos casos y desconociéndola en otros; f) o en el caso de que un órgano judicial incurra en una negativa manifiesta del deber de acatamiento de la doctrina del Tribunal Constitucional (art. 5 de la Ley Orgánica del Poder Judicial: LOPJ); g) o, en fin, cuando el asunto suscitado, sin estar incluido en ninguno de los supuestos anteriores, trascienda del caso concreto porque plantee una cuestión jurídica de relevante y general repercusión social o económica o tenga unas consecuencias políticas generales, consecuencias que podrían concurrir, sobre todo, aunque no exclusivamente, en determinados amparos electorales o parlamentarios". STC 155/2009 de 25 de junio, Fj 2.

61 El concepto de "especial trascendencia constitucional" es considerado por Ortega Gutiérrez como un concepto jurídico indeterminado. Al respecto Ortega Gutiérrez (2010) pp. 497 ss. Para Pulido Quecedo, la determinación del concepto de "especial trascendencia constitucional" que realiza el Tribunal Constitucional es de agradecerse, aunque parece reflejar una función cuasi-legislativa. Pulido Quecedo (2009) pp. 3 ss.

62 STC 155/2009 de 25 de junio, Fj 2. 
margen de maniobra bastante amplio del que goza para establecer si un amparo trata o no una cuestión de "especial trascendencia constitucional" 63.

\section{CONCLUSIONES}

De la historia de la jurisdicción apelada de la Corte Suprema de los Estados Unidos puede obtenerse una importante lección para los debates que se abren en otros países acerca de cómo enfrentar la sobrecarga de trabajo de sus tribunales de última instancia con competencia para ejercer el control de constitucionalidad. Para abordar esta problemática es necesario previamente replantearse cuál es la función primordial del tribunal que padece una sobrecarga de trabajo y decidir concentrarse solo o principalmente en ella. Clarificada esta cuestión es más sencillo establecer cuál es el mejor modo para llevar a cabo tal función.

La transformación en discrecional de prácticamente toda la jurisdicción de la Corte Suprema de los Estados Unidos y la particular forma en que dicho tribunal ejerce su jurisdicción están claramente orientadas a que pueda cumplir su principal función de aclarar el derecho federal, ofreciendo una interpretación final de dicho derecho.

No obstante, además de esta lección también puede ser útil tener en cuenta que una jurisdicción discrecional como la concedida a la Corte Suprema de los Estados Unidos tiene el desafío de lograr ser aceptada como legítima o no arbitraria. Si bien conseguir esto es en gran medida responsabilidad del tribunal, puede ser útil para esto tener en cuenta otras experiencias sobre cómo limitar parte de dicha discrecionalidad.

\section{BIBLIOGRAFÍA CITADA}

Baude, William (2015): "Foreword: The Supreme Court's Shadow Docket", New York University Journal of Law and Liberty, vol. $9 \mathrm{~N}^{\circ} 1$ : pp. 1-46.

BianchI, Alberto (1994): Jurisdicción y procedimientos en la Corte Suprema de los Estados Unidos (Buenos Aires, Ábaco).

Calabresi, Steven G. y Presser, David C. (2006): "Reintroducing Circuit Riding: A Timely Proposal", Minnesota Law Review, vol. N 90: pp. 1386-1416.

Carmona Cuenca, Encarnación (2005): La crisis del recurso de amparo (Madrid, Universidad de Alcalá).

Carrington, Paul D. y Cramton, Roger C. (2009): "Judicial Independence in Excess: Reviving the Judicial Duty of the Supreme Court", Cornell Law Review, vol. $94 \mathrm{~N}^{\circ}$ 3: pp. 587-636.

Etcheverry, Juan B. (2015): “Cómo ha resuelto el problema de la sobrecarga de trabajo el Tribunal Constitucional de España”, Cuestiones Constitucionales, No 32: pp. 141-155.

García RocA, Javier (2003): "La cifra del amparo constitucional y su reforma". Disponible en : http://www.google.com.ar/\#hl=en\&tbo=d\&sclient=psyab\&q=J+garc\%C3\%ADa+r oca+la+cifra+del+amparo\&oq $=\mathrm{J}+$ garc $\% \mathrm{C} 3 \% \mathrm{ADa}+\mathrm{roca}+\mathrm{la}+\mathrm{cifra}+\mathrm{del}+\mathrm{amparo} \& g s \mathrm{l}=$ serp

${ }^{63}$ Sobre cómo el Tribunal Constitucional de España resuelve el problema de la sobrecarga de trabajo de un modo menos radical ETCHEVERRY (2014). 


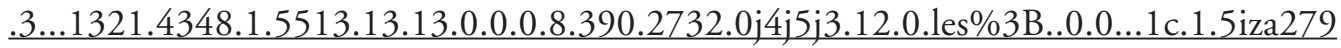
$2 \mathrm{ffg} \& \mathrm{pbx}=1 \& \mathrm{bav}=$ on. 2 ,or.r gc.r pw.r qf. $\& \mathrm{fp}=\mathrm{f} 68 \mathrm{c} 38 \mathrm{ad} 5 \mathrm{e} 0 \mathrm{dff} 73 \& \mathrm{bpcl}=40096503 \& \mathrm{~b}$ $\underline{\mathrm{iw}=1093 \& \mathrm{bih}=538}$. Fecha de consulta: 18 de diciembre de 2012.

Hartnett, Edward A. (2000): "Questioning Certiorari: Some Reflections Seventy-five Years After the Judges' Bill”, Columbia Law Review, vol. № 100: pp. 1643-1738.

Hernández Ramos, Mario (2009): El nuevo trámite de admisión del recurso de amparo constitucional (Madrid, Reus).

Hobson, Charles F. (2006): "Defining the Office: John Marshall as Chief Justice", University of Pennsylvania Law Review, vol. 154: pp. 1421-1461.

Lazarus, Richard J. (2009): "Docket Capture at the High Court", Yale Law Journal Online, vol. 119: pp. 89-97.

Le Sueur, Andrew y Cornes, Richard (2000): "What Do the Top Courts Do?", Current Legal Problems, vol. $53 \mathrm{~N}^{\circ} 1$ : pp. 53-97.

New York Times (26/09/2008) Disponible en: http://www.nytimes.com/2008/09/26/ washington/26memo.html? r=0. Fecha de consulta: 15 de diciembre de 2013.

Meriwether Cordray, Margaret y Cordray, Richard (2004): "The Philosophy of Certiorari: jurisprudential Considerations in Supreme Court Case Selection”, Washington University Law Quarterly, vol. 82 N $^{\circ}$ : pp. 389-452.

Meriwether Cordray, Margaret y Cordray, Richard (2008): "Strategy in Supreme Court Case Selection: The Relationship Between Certiorari and the Merits", Ohio State Law Journal, vol. $69 \mathrm{~N}^{\circ} 1$ : pp. 1-51.

Meriwether Cordray, Margaret y Cordray, Richard (2009): "Setting the Social Agenda: Deciding to Review High-Profile Cases at the Supreme Court", University Kansas Law Review, vol. 57: pp. 313-369.

Ortega Gutiérrez, David (2010): "La especial trascendencia constitucional como concepto jurídico indeterminado. De la reforma de 2007 de la LOTC a la STC 155/2009, de 25 de junio", Teoría y realidad constituciona,l vol. 25: pp. 497-513.

Peppers, Todd C. (2006): Courtiers of the Marble Palace: The Rise and Influence of the Supreme Court Law Clerk (Stanford, Stanford University Press).

Perry, Hersel W. (Jr.) (1994): Deciding to Decide. Agenda Setting in the United States Supreme Court (Cambridge, Harvard University Press).

Perry, Hersel W. (Jr.) (2005): “Certiorari”, en Hall, Kermit L. (edit.), The Oxford Companion to the Supreme Court (New York, Oxford University Press, segunda edición) pp. 154-155.

Perry, Hersel W. (Jr.) (2010): "Access to Justice: Procedure, Polity, and Politics", Bond Law REVIEW, VOL. 22 N³: pp. 191-211.

Pulido Quecedo, Manuel (2009): "El requisito de 'la especial trascendencia constitucional en el recurso de amparo", Revista Aranzadi Doctrinal, vol. 6: pp. 73-78.

Rehnquist, William H. (1987): The Supreme Court, How it was, How it is (New York, William Morrow \& Co).

SACRITÁn, Estela B. (2003): “Acceso a la información judicial”, en SACritán, Estela B. (Dir.) Manual de Jurisprudencia y Doctrina (Buenos Aires, La Ley) pp. 447-468. 
SaCritÁn, Estela B. (2009): "El rol docente de la Corte Suprema (En torno al art. 11 de la Acordada 4/2007)”, La Ley, vol. F: pp. 1102-1113.

Shapiro, Stephen M., (1998): "Certiorari Practice: The Supreme Court's Shrinking Docket”, Litigation, vol. $24 \mathrm{~N}^{\circ}$ 3: pp. 25-33.

Stern, Robert L.; Gressman, Eugene y Shapiro, Stephen M. (1986): Supreme Court Practice (Washington D.C., The Bureau of National Affairs, sexta edición).

SternberG, Jonathan (2008): "Deciding Not to Decide: The Judiciary Act of 1925 and the Discretionary Court”, The Journal of Supreme Court History, vol. N 33: pp. 1-16.

Sturley, Michael. F. (2005): "Certiorari Pool”, en Hall, Kermit L., The Oxford Companion to the Supreme Court (New York, Oxford University Press, segunda edición) pp. 155-156.

Tyler, Amanda L. (2009): "Setting the Supreme Courts Agenda: Is There a Place for Certification?", George Washington Law Review vol. 78 N 6: pp. 1310-1328.

Watts, Kathryn A. (2011): "Constraining Certiorari Using Administrative Law Principles”, University of Pennsylvania Law Review, vol. $160 \mathrm{~N}^{\circ}$ 1: pp. 1-68.

Wheeler, Russell R. y Harrinson, Cynthia (2005): Creating the Federal Judicial System (Washington D.C., Federal Judicial Center, tercera edición).

Yablon, Robert M. (2014): “Justice Sotomayor and the Supreme Court's Certiorari Process", The Yale Law Journal Forum, vol. 123: pp. 551-564.

\section{NORMAS CITADAS}

Código Federal de los Estados Unidos, última modificación de 2012. Disponible en: http://uscodebeta.house.gov/browse/prelim@title28/part4/chapter81\&editio $\mathrm{n}=$ prelim. Fecha de consulta: 5 de diciembre de 2013.

Ley Orgánica del Tribunal Constitucional de España: Disponible en : http://www. tribunalconstitucional.es/es/tribunal/normasreguladoras/Lists/NormasRegPDF/Normas\%20Reguladoras/leyorgtrib.pdf. Fecha de consulta: 15 de septiembre de 2013.

Regla 10 de la Corte Suprema de los Estados Unidos, Disponible en:www. supremecourt.gov/ctrules/2010RulesoftheCourt.pdfwww.supremecourt.gov/ ctrules/2010RulesoftheCourt.pdf. Fecha de consulta: 5 de septiembre de 2013.

Regla 12 de la Corte Suprema de los Estados Unidos. Disponible en: www. supremecourt.gov/ctrules/2010RulesoftheCourt.pdfwww.supremecourt.gov/ ctrules/2010RulesoftheCourt.pdf. Fecha de consulta: 5 de septiembre de 2013.

\section{JURISPRUDENCIA CITADA}

COHENS V. VIRGINIA, 19 U.S. 264, 404 (1821).

COUNTY OF SONOMA V. ISBELL, 439 U.S. 996 (1978).

ILLINOIS V. GRAY, 435 U.S. 1013 (1978).

STC 155/2009 de 25 de junio. 